\title{
THE SEGRE CUBIC AND BORCHERDS PRODUCTS
}

\author{
SHIGEYUKI KONDŌ
}

\begin{abstract}
We shall construct a 5-dimensional linear system of holomorphic automorphic forms on a 3-dimensional complex ball by applying Borcherds theory of automorphic forms. We shall show that this linear system gives the dual map from the Segre cubic 3-fold to the Igusa quartic 3-fold.
\end{abstract}

\section{INTRODUCTION}

The main purpose of this note is to give an application of the theory of automorphic forms on bounded symmetric domains of type IV due to Borcherds [5], [6]. We consider the Segre cubic 3-fold $X$ which is a hypersurface of $\mathbf{P}^{4}$ of degree 3 with ten nodes. The symmetry group $\mathfrak{S}_{6}$ of degree 6 acts on $X$ as projective transformations. It is known that the Segre cubic $X$ is isomorphic to the SatakeBaily-Borel compactification of an arithmetic quotient of a 3-dimensional complex ball $\mathcal{B}$ associated to a hermitian form of signature $(1,3)$ defined over the Eisenstein integers ([11], [12], [13]). The complex ball $\mathcal{B}$ can be embeded into a bounded symmetric domain $\mathcal{D}$ of type IV and of dimension 6. By applying Borcherds' theory of automorphic forms on bounded symmetric domains of type IV, we can construct a 5-dimensional linear system of holomorphic automorphic forms of weight 6 . We shall show that this linear system gives the dual map from the Segre cubic $X$ to its dual Igusa quartic 3-fold.

B. van Geemen [10] and B. Hunt [11] observed that both the Segre cubic 3-fold and the Igusa quartic 3-fold are birational to the moduli of ordered 6 points on the projective line. By taking the triple cover of $\mathbf{P}^{1}$ branched along 6 points we get a curve of genus 4 with an automorphism of order 3. One can consider that the Segre cubic is a compactification of the moduli of such curves. On the other hand, by taking the double cover of $\mathbf{P}^{1}$ branched along 6 points, we get a hyperelliptic curve of genus 2. The Igusa quartic is the Satake compactification of an arithmetic quotient of the Siegel space of degree 2 (J. Igusa [14], page 397). It is classically known that the dual of the Segre cubic is isomorphic to the Igusa quartic (Baker [4], Chap. V). We will give an interpretation of this ball quotient as the moduli space of some $K 3$ surfaces with an automorphism of order 3 which are obtained from 6 points on the projective line.

We use an idea of Allcock, Freitag [3] to construct a linear system of automorphic forms. In [3], they consider a 4-dimensional complex ball defined over the Eisenstein integers and construct a 10-dimensional linear system of automorphic forms. An arithmetic quoteint of the 4-dimensional complex ball is birational to the moduli space of marked cubic surfaces. Our complex ball $\mathcal{B}$ appears as a subcomplex ball of Allcock, Freitag's one, and hence one can restrict Allcock, Freitag's linear system to $\mathcal{B}$. However in this note, instead of using their linear system, we apply Borcherds theory directly to our situation and get a linear system on $\mathcal{B}$.

The plan of this note is as follows. In $\S 2$, we recall the Segre cubic $X$ and some divisors on $X$. In section 3 , we mention the complex ball $\mathcal{B}$, the bounded symmetric domain $\mathcal{D}$ and Heegner divisors

Partially supported by JSPS Grant-in-Aid (S), No 22224001, No 19104001, Japan. 
on them. In section 4, we recall the Weil representation and calculate its character. In section 5, we shall show that there exist holomorphic automorphic forms on the complex ball $\mathcal{B}$ of weight 45,5 with known zeros. These forms will be used to determine the zeros of a member of a 5-dimensional linear system of automorphic forms on $\mathcal{B}$. In section 6 , we construct a 5-dimensional linear system of automorphic forms and show that this linear system gives the dual map of the Segre cubic.

\section{The SEgRE CUBiC THREEFOLD}

In this note we consider the variety $X$ called the Segre cubic which is defined by

$$
X: \sum_{i=1}^{6} x_{i}=0, \sum_{i=1}^{6} x_{i}^{3}=0
$$

in $\mathbf{P}^{5}$. Obviously the symmetric group $\mathfrak{S}_{6}$ of degree 6 acts on $X$ projectively. $X$ has ten nodes which are $\mathfrak{S}_{6}$-orbits of $(1: 1: 1:-1:-1:-1)$. A linear section of $X$ given by $x_{i}+x_{j}=0$ is the union of three projective planes given by

$$
\begin{aligned}
& x_{i}+x_{j}=0, x_{k}+x_{l}=0, x_{m}+x_{n}=0 ; \\
& x_{i}+x_{j}=0, x_{k}+k_{m}=0, x_{l}+x_{n}=0 ; \\
& x_{i}+x_{j}=0, x_{k}+x_{n}=0, x_{l}+x_{m}=0,
\end{aligned}
$$

respectively where $\{i, j, k, l, m, n\}=\{1,2,3,4,5,6\}$. On the other hand a linear section of $X$ given by $x_{i}-x_{j}=0$ is an irreducible cubic surface containing four nodes of $X$. This irreducible cubic surface with four node is projectively unique and is called Cayley cubic surface. Thus we have 15 Cayley cubics and 15 planes on the Segre cubic $X$. It is known that the dual of $X$ is a quartic 3-fold $Y$ in $\mathbf{P}^{4}$ called the Igusa quartic ([14]). The dual map $d: X \rightarrow Y$ is defined on $X$ except ten nodes and is birational. It is given by a linear system of quadrics through ten nodes. For more details of these facts, we refer the reader to [13], Chap. 3.

\section{A COMPLEX BALL QUOTIENT}

It is known that the Segre cubic $X$ is isomorphic to the Satake-Baily-Borel compactification of an arithmetic quotient of a 3-dimensional complex ball by a certain arithmetic subgroup ([12], Theorem 1; [13], Chap. 3, 3.2.3). In this section we recall this fact.

\subsection{A complex ball. Let}

$$
\mathcal{E}=\mathbf{Z}[\omega], \omega=\frac{-1+\sqrt{-3}}{2}
$$

be the ring of Eisenstein integers. Consider the hermitian lattice

$$
\Lambda=\mathcal{E}^{1,3}=\mathcal{E} \oplus \mathcal{E} \oplus \mathcal{E} \oplus \mathcal{E}
$$

with the hermitian form

$$
h(x, y)=x_{0} \bar{y}_{0}-x_{1} \bar{y}_{1}-x_{2} \bar{y}_{2}-x_{3} \bar{y}_{3} .
$$

We denote by $\mathcal{B}$ the complex ball of dimension 3 defined by

$$
\mathcal{B}=\left\{x \in \mathbf{P}\left(\Lambda \otimes_{\mathcal{E}} \mathbf{C}\right): h(x, x)>0\right\} .
$$


Let $\Gamma=\operatorname{Aut}(\Lambda)$ be an arithmetic subgroup of the unitary group $U(3,1 ; \mathbf{Q}(\sqrt{-3}))$ with respect to the hermitian form $h($,$) . Obviously \Gamma$ naturally acts on $\mathcal{B}$. Under the isomorphism

$$
\mathcal{E} / \sqrt{-3} \mathcal{E} \simeq \mathbf{F}_{3}
$$

the hermitian form $h$ induces a quadratic form $q$ on $\Lambda / \sqrt{-3} \Lambda$ over $\mathbf{F}_{3}$. We define a subgroup $\Gamma(\sqrt{-3})$ of $\Gamma$ by

$$
\Gamma(\sqrt{-3})=\operatorname{Ker}\{\Gamma \longrightarrow \mathrm{O}(q)\}
$$

Let $L$ be the real lattice corresponding to $\Lambda$ with the symmetric bilinear form

$$
\langle x, y\rangle=h(x, y)+h(y, x) .
$$

Then $L \cong A_{2} \oplus A_{2}(-1)^{3}$ where $A_{2}$ is a root lattice of rank 2 , that is, a positive definite lattice of rank 2 defined by the matrix $\left(\begin{array}{cc}2 & -1 \\ -1 & 2\end{array}\right)$ and $A_{2}(-1)$ is the negative definite lattice of rank 2 defined by $\left(\begin{array}{cc}-2 & 1 \\ 1 & -2\end{array}\right)$. The action of $\omega$ on $\Lambda$ induces an isometry $\iota$ of $L$ of order 3 without non-zero fixed points. We denote by $L^{*}$ the dual of $L: L^{*}=\operatorname{Hom}(L, \mathbf{Z})$. Note that $A_{2}^{*} / A_{2} \cong \mathbf{F}_{3}$. Let $A_{L}=L^{*} / L \cong\left(\mathbf{F}_{3}\right)^{4}$ and let $q_{L}: A_{L} \rightarrow \mathbf{Q} / 2 \mathbf{Z}$ be the discriminant quadratic form of $L$ defined by $q_{L}(x+L)=\langle x, x\rangle+2 \mathbf{Z}$. The form $q_{L}$ coincide with $q$, up to scales, under the isomorphism

$$
\Lambda / \sqrt{-3} \Lambda \cong L^{*} / L
$$

We denote by $\mathrm{O}(L)$ the orthogonal group of $L$ and by $\tilde{\mathrm{O}}(L)$ the kernel of the natural map $\mathrm{O}(L) \rightarrow$ $\mathrm{O}\left(q_{L}\right)$. Then the group $\Gamma$ is naturally isomorphic to the subgroup $\mathrm{O}(L, \iota)$ of $\mathrm{O}(L)$ consisting of isometries commuting with $\iota$. Under this isomorphism the subgroup $\Gamma(\sqrt{-3})$ corresponds to $\mathrm{O}(L, \iota) \cap$ $\tilde{\mathrm{O}}(L)$.

Conversely, first consider the lattice $L$ with an automorphism $\iota$ of order 3 without non-zero fixed points. Then we can consider $L$ as $\mathbf{Z}[\omega]$-module by the action $\omega \cdot x=\iota(x)$. The hermitian form $h$ is given by

$$
h(x, y)=\frac{1}{2}\left\{\frac{\sqrt{-3}}{3}\langle 2 \iota(x)+x, y\rangle+\langle x, y\rangle\right\}
$$

Define

$$
\mathcal{D}=\{v \in \mathbf{P}(L \otimes \mathbf{C}):\langle v, v\rangle=0,\langle v, \bar{v}\rangle>0\}
$$

Then $\mathcal{D}$ is a disjoint union of two copies of a bounded symmetric domain of type IV and of dimension 6. Consider the action of $\iota$ on $L \otimes \mathbf{C}$. Since $\iota$ is defined over $\mathbf{Z}$ and has no non-zero fixed vectors, the eigen-spaces $V_{\omega}, V_{\bar{\omega}}$ with the eigen-value $\omega, \bar{\omega}$ respectively are isomorphic to $\mathbf{C}^{4}$. Moreover the restriction $\langle v, \bar{v}\rangle$ on $V_{\omega}$ is a hermitian form of signature $(1,3)$. Note that $\langle v, v\rangle=0$ for any vector $v$ in $V_{\omega}$ or $V_{\bar{\omega}}$ because $\langle v, v\rangle=\langle\iota(v), \iota(v)\rangle=\langle\omega v, \omega v\rangle$ or $\langle v, v\rangle=\langle\bar{\omega} v, \bar{\omega} v\rangle$. Let $i: \Lambda \rightarrow \Lambda \otimes_{\mathbf{Z}} \mathbf{C}=L \otimes \mathbf{C}$ be the inclusion map and let $p: L \otimes \mathbf{C} \rightarrow V_{\omega}$ be the projection. For any $\xi \in L$, write $\xi=\xi_{\omega}+\xi_{\bar{\omega}}$ as an element in $V_{\omega} \oplus V_{\bar{\omega}}$. Then we can easily see that

$$
h(\xi, \xi)=\left\langle\xi_{\omega}, \bar{\xi}_{\omega}\right\rangle
$$

Hence the map $p \circ i: \Lambda \rightarrow V_{\omega}$ is an isometry which induces an isomorphism from $\mathcal{B}$ to the subdomain

$$
\mathcal{D} \cap \mathbf{P}\left(V_{\omega}\right)=\left\{v \in \mathbf{P}\left(V_{\omega}\right):\langle v, \bar{v}\rangle>0\right\}
$$

of $\mathcal{D}$. Thus the complex ball $\mathcal{B}$ can be embedded into $\mathcal{D}$. 
3.2. Roots and reflections. Following to [3], we recall roots and reflections of the hermitian lattice $\Lambda$. A vector $a \in \Lambda$ is called a short root (resp. long root) if $h(a, a)=-1$ (resp. $h(a, a)=-2$ ). For a short root or long root $a$, consider the following isometry $r_{a, \zeta}$ of $\Lambda$ with respect to $h$ :

$$
r_{a, \zeta}: x \rightarrow x-(1-\zeta) \frac{h(a, v)}{h(a, a)} a .
$$

If $a$ is a short root and $\zeta$ is a primitive third root of unity $\omega, r_{a, \omega}$ is an isometry of $\Lambda$ of order three sending $a$ to $\omega a$. We call $r_{a, \omega}$ a trireflection. If $a$ is a short root or long root, and $\zeta=-1$, then $r_{a,-1}$ is a reflection in $\Gamma$ which is an isometry of order two sending $a$ to $-a$.

For a short root $a$ in $\Lambda$, denote by $r$ the corresponding $(-2)$-vector in $L$. Then the trireflection $r_{a, \omega}$ induces an isometry

$$
s_{r} \circ s_{\iota(r)}: x \rightarrow x+\langle x, r\rangle r+\langle x, \iota(r)\rangle r+\langle x, \iota(r)\rangle \iota(r)
$$

of $L$ where $s_{r}: x \rightarrow x+\langle x, r\rangle r$ is a reflection associated to $r$. On the other hand, $r_{a,-1}$ induces an isometry of $L$ :

$$
x \rightarrow x+2\left\langle\frac{r+2 \iota(r)}{3}, x\right\rangle \iota(r)+2\left\langle\frac{2 r+\iota(r)}{3}, x\right\rangle r .
$$

For a long root $a \in \Lambda$, denote by $r$ the corresponding $(-4)$-vector in $L$. Then $r_{a,-1}$ induces an isometry of $L$ :

$$
x \rightarrow x+\left\langle\frac{r+2 \iota(r)}{3}, x\right\rangle \iota(r)+\left\langle\frac{2 r+\iota(r)}{3}, x\right\rangle r .
$$

For $a \in \Lambda$, we denote by $\bar{a}$ the image of $a$ in $\Lambda / \sqrt{-3} \Lambda$. We call the images of short roots (resp. long roots) in $\Lambda / \sqrt{-3} \Lambda$ the short roots (resp. long roots), too. We also denote by $\bar{r}_{a, \zeta}$ the isometry on $\Lambda / \sqrt{-3} \Lambda$ induced by $r_{a, \zeta}$. Note that if $a$ is a short root, then $r_{a, \omega}$ is contained in $\Gamma(\sqrt{-3})$, that is, $\bar{r}_{a, \omega}$ acts trivially on $\Lambda / \sqrt{-3} \Lambda$. On the other hand, $\bar{r}_{a,-1}$ acts on $\Lambda / \sqrt{-3} \Lambda$ as a reflection associated to $\bar{a}$.

3.3. Lemma. (1) The group $\Gamma$ acts transitively on the primitive isotropic vectors, on the short roots and on the long roots, respectively.

(2) Let $a_{1}, a_{2}$ be two isotropic vectors, or two short roots, or two long roots. Then $a_{1}$ and $a_{2}$ are equivalent under $\Gamma(\sqrt{-3})$ if and only if their images in $\Lambda / \sqrt{-3} \Lambda$ coincide.

(3) The number of non-zero isotropic vectors, short roots or long roots in $\Lambda / \sqrt{-3} \Lambda$ is 20 , 30 or 30 respectively.

(4) The map $\Gamma \rightarrow O(q)$ is surjective and $\Gamma / \Gamma(\sqrt{-3}) \simeq O(q) \simeq \mathfrak{S}_{6} \times \mathbb{Z} / 2 \mathbb{Z}$.

Proof. In case of the hermitian lattice $\mathcal{E}^{1,4}$, Allcock, Carlson, Toledo proved the same assertion (1) ([2], Theorems 7.21, 11.13), and Allcock, Freitag ([3], Proposition 2.1) proved the assertions (2), (3). The same proof works in our case $\mathcal{E}^{1,3}$. The last assertion is a well know. For example, see [7], page 4.

3.4. Ball quotient and Heegner divisors. We denote a vector $\alpha \in A_{L}=\left(\mathbf{F}_{3}\right)^{4}$ by $\alpha=\left(x_{1}, x_{2}, x_{3}, x_{4}\right)$ where $x_{i} \in \mathbf{F}_{3}$ is in the $i$-th factor of $L^{*} / L=A_{2}^{*} / A_{2} \oplus\left(A_{2}(-1)^{*} / A_{2}(-1)\right)^{\oplus 3}$. Then an elementary calculation shows the following: 
3.5. Lemma. (i) $A_{L}$ consists of the following 81 vectors:

Type (00): 0;

Type $(0): \alpha \neq 0, q_{L}(\alpha)=0, \# \alpha=20$,

$\alpha=( \pm 1, \pm 1,0,0),( \pm 1,0, \pm 1,0),( \pm 1,0,0, \pm 1),(0, \pm 1, \pm 1, \pm 1)$;

Type $(1): q_{L}(\alpha)=-4 / 3, \# \alpha=30$,

$\alpha=( \pm 1,0,0,0),( \pm 1, \pm 1, \pm 1, \pm 1),(0, \pm 1, \pm 1,0),(0, \pm 1,0, \pm 1),(0,0, \pm 1, \pm 1)$;

Type $(2): q_{L}(\alpha)=-2 / 3, \# \alpha=30$,

$\alpha=(0, \pm 1,0,0),(0,0, \pm 1,0),(0,0,0, \pm 1),( \pm 1, \pm 1, \pm 1,0),( \pm 1, \pm 1,0, \pm 1),( \pm 1,0, \pm 1, \pm 1)$.

3.6. Lemma. Under the canonical isomorphism $\Lambda / \sqrt{-3} \Lambda \cong A_{L}=L^{*} / L$, the set of short roots (resp. long roots) in $\Lambda / \sqrt{-3} \Lambda$ correspond to the set of vectors of norm $-2 / 3$ (resp. vectors of norm $-4 / 3)$ in $A_{L}$. Also the set of isotropic vectors in $\Lambda / \sqrt{-3} \Lambda$ correspond to the set of isotropic vectors in $A_{L}$.

Let $\alpha$ be a non-isotropic vector in $A_{L}$. For a given $n \in \mathbf{Z}, n<0$, we consider a Heegner divisor $\mathcal{D}_{\alpha, n}$ which is the union of the orthogonal complements $r^{\perp}$ in $\mathcal{D}$ where $r$ varies over the vectors in $L^{*}$ satisfying $\langle r, r\rangle=n$ and $r \bmod L=\alpha$. Obviously $r^{\perp}$ is a bounded symmetric domain of type IV and of dimension 5. In case $q_{L}(\alpha)=-2 / 3$ (resp. $\left.q_{L}(\alpha)=-4 / 3\right)$ and $n=-2 / 3($ resp. $n=-4 / 3)$, we denote $\mathcal{D}_{\alpha,-2 / 3}$ (resp. $\left.\mathcal{D}_{\alpha,-4 / 3}\right)$ by $\mathcal{D}_{\alpha}$ for simplicity and call it $(-2 / 3)$-Heegner divisor (resp. (-4/3)-Heegner divisor).

3.7. Proposition. The Segre cubic $X$ is isomorphic to the Satake-Baily-Borel compactification $\overline{\mathcal{B}} / \Gamma(\sqrt{-3})$ of the quotient $\mathcal{B} / \Gamma(\sqrt{-3})$ which is, set theoretically, the union of $\mathcal{B} / \Gamma(\sqrt{-3})$ and ten cusps corresponding to ten non-zero isotoropic vectors in $A_{L} /\{ \pm 1\}$. These ten cusps correspond to ten nodes of the Segre cubic $X$.

Proof. The assertion follows from [13], §3.2.

Also $\overline{\mathcal{B}} / \Gamma(\sqrt{-3})$ contains some divisors called Heegner divisors. Let $\alpha$ be a short root in $\Lambda / \sqrt{-3} \Lambda$. Let $a$ be a short root in $\Lambda$ with $a \bmod \sqrt{-3} \Lambda=\alpha$. We denote by $a^{\perp}$ the orthogonal complement of $a$ in $\mathcal{B}$ which is a complex ball of dimension 2. Let

$$
\mathcal{H}_{\alpha}=\bigcup_{a} a^{\perp}
$$

where $a$ moves on the set of all short roots satisfying $a \bmod \sqrt{-3} \Lambda=\alpha$. The image of $\mathcal{H}_{\alpha}$ in $\overline{\mathcal{B}} / \Gamma(\sqrt{-3})$ is denoted by $\overline{\mathcal{H}}_{\alpha}$ and is called a $(-1)$-Heegner divisor. There exist $15(-1)$-Heegner divisors $\overline{\mathcal{H}}_{\alpha}$ corresponding to 15 short roots $\alpha \in(\Lambda / \sqrt{-3} \Lambda) /\{ \pm 1\}, q(\alpha)=-1$.

Similarly we can define $15(-2)$-Heegner divisors $\overline{\mathcal{H}}_{\alpha}$ corresponding to 15 long roots $\alpha \in(\Lambda / \sqrt{-3} \Lambda) /\{ \pm 1\}$, $q(\alpha)=-2$.

Finally we compare the Heegner divisors in $\mathcal{D}$ and in $\mathcal{B}$. Let $r \in L^{*}$ be a $(-2 / 3)$ - or $(-4 / 3)$-vector. Then both $\iota(r)$ and $\iota^{2}(r)$ are $(-2 / 3)$ - or $(-4 / 3)$-vectors and $r \bmod L=\iota(r) \bmod L=\iota^{2}(r) \bmod L$. Note that $r^{\perp}, \iota(r)^{\perp}$ and $\iota^{2}(r)^{\perp}$ in $\mathcal{D}$ are different, but their restrictions to $\mathcal{B}$ are the same. Thus we have 


\subsection{Lemma.}

$$
\mathcal{D}_{\alpha} \cap \mathcal{B}=3 \mathcal{H}_{\alpha}
$$

where we identify $(-2 / 3)$ - (resp. (-4/3)-) vectors in $A_{L}$ and short roots (resp. long roots) in $\Lambda / \sqrt{-3} \Lambda$.

3.9. Interpretation via $K 3$ surfaces. The complex ball quotient $\mathcal{B} / \Gamma(\sqrt{-3})$ can be considered as the moduli space of lattice polarized $K 3$ surfaces. The following is essentially given in [8]. Let $N=U \oplus E_{6}(-1) \oplus A_{2}(-1)^{\oplus 3}$. Then $N$ can be primitively embedded into the $K 3$ lattice $M=$ $U^{\oplus 3} \oplus E_{8}(-1)^{\oplus 2}$ whose orthogonal complement is isomorphic to $L=A_{2} \oplus A_{2}(-1)^{\oplus 3}$. Here $A_{m}, E_{k}$ are positive definite root lattices defined by the Cartan matrix of type $A_{m}, E_{k}$, and for a lattice $(L,\langle\rangle$, we denote by $L(-1)$ is the lattice $(L,-\langle\rangle$,$) . In the following we consider N$ and $L$ as sublattices of $M$. The isometry $\iota$ of $L$ of order 3 acts trivially on $L^{*} / L$ and hence it can be extended to an isometry $\tilde{\iota}$ of $M$ acting trivially on $N$. Let $\omega \in \mathcal{B}$ with the property $\omega^{\perp} \cap M=N$. Let $S$ be a $K 3$ surface and let $\alpha_{S}: H^{2}(S, \mathbf{Z}) \rightarrow M$ be an isometry satisfying $\left(\alpha_{S} \otimes \mathbf{C}\right)\left(\omega_{S}\right)=\omega$ where $\omega_{S}$ is a holomorphic 2-form on $S$. By definition the Picard lattice of $S$ is isomorphic to $N$. Note that the isometry $\tilde{\iota}$ preserves $\omega_{S}$ and acts trivially on the Picard lattice. It now follows from the Torelli type theorem for $K 3$ surface that $\tilde{\iota}$ can be represented by an automorphism $\sigma$ on $S$ of order 3 . Thus an open set of $\mathcal{B} / \Gamma(\sqrt{-3})$ is the moduli of such pairs $(S, \sigma)$ of $K 3$ surfaces $S$ with an automorphism $\sigma$ of order 3 .

In the following, we shall show that $S$ is canonically obtained from six points on $\mathbf{P}^{1}$. Let $Q=$ $\mathbf{P}^{1} \times \mathbf{P}^{1}$. Let $\left(u_{0}: u_{1}\right),\left(v_{0}: v_{1}\right)$ be homogeneous coordinates of the first and the second factor of $Q$. Let $p_{1}, \ldots, p_{6}$ be distinct six points on $\mathbf{P}^{1}$. Consider the divisors on $Q$ defined by

$$
L_{i}=\mathbf{P}^{1} \times\left\{p_{i}\right\}(1 \leq i \leq 6), D_{0}=\{0\} \times \mathbf{P}^{1}, D_{1}=\{1\} \times \mathbf{P}^{1}, D_{\infty}=\{\infty\} \times \mathbf{P}^{1} .
$$

Let $\tilde{Q} \rightarrow Q$ be the blow ups of the 18 points on $Q$ which are the intersection of $L_{1}, \ldots, L_{6}$ and $D_{0}, D_{1}, D_{\infty}$. We denote by the $\tilde{L}_{1}, \ldots, \tilde{L}_{6}, \tilde{D}_{0}, \tilde{D}_{1}$ or $\tilde{D}_{\infty}$ the strict transform of $L_{1}, \ldots, L_{6}, D_{0}, D_{1}$ or $D_{\infty}$ respectively. Let $\pi: \tilde{X} \rightarrow \tilde{Q}$ be the triple covering of $\tilde{Q}$ branched along $\tilde{L}_{1}+\cdots+\tilde{L}_{6}+$ $\tilde{D}_{0}+\tilde{D}_{1}+\tilde{D}_{\infty}$. Then $\pi^{-1}\left(\tilde{L}_{i}\right)$ is a $(-1)$-curve. Let $\tilde{X} \rightarrow S$ be the contraction of $\pi^{-1}\left(\tilde{L}_{i}\right)$ to the points $q_{i}$. We can easily see that $S$ is a K3 surface. The projection from $Q$ to the second factor $\mathbf{P}^{1}$ induces an elliptic fibration $p: S \rightarrow \mathbf{P}^{1}$ which has six singular fibers of type IV in the notation of Kodaira and three sections. Here three components of the singular fiber of type IV over $p_{i}$ correspond to three exceptional curves over the three intersection points of $L_{i}$ and $D_{0}, D_{1}, D_{\infty}$ and three sections correspond to $D_{0}, D_{1}, D_{\infty}$. The classes of components of fibers and a section generate a sublattice of the Picard lattice $\operatorname{Pic}(S)$ isomorphic to $U \oplus A_{2}(-1)^{\oplus 6}$. By adding other two sections, we have a sublattice in $\operatorname{Pic}(S)$ isomorphic to $N=U \oplus E_{6}(-1) \oplus A_{2}(-1)^{\oplus 3}$. The covering transformation of $\tilde{S} \rightarrow \tilde{Q}$ induces an automorphism $\sigma$ of $S$ of order 3 . Note that the set of fixed points of $\sigma$ consists of six isolated points $q_{1}, \ldots, q_{6}$ and three sections. Since $\sigma$ has a fixed curve as its fixed points, $\sigma^{*}\left(\omega_{S}\right)=\zeta_{3} \omega_{S}$ where $\omega_{S}$ is a non-zero holomorphic 2-form on $S$ and $\zeta_{3}$ is a primitive cube root of unity. Thus we have a pair $(S, \sigma)$ of a $K 3$ surface and an automorphism of order 3 . This $K 3$ surface appears as a degeneration of $K 3$ surfaces associated to a smooth cubic surface given in [8].

Next we consider the case two points among 6 points coincide. In this case, similarly, we have an elliptic $K 3$ surface $S^{\prime}$ with one singular fiber of type $\mathrm{VI}^{*}$, four singular fibers of type VI and three sections. The Picard lattice of $S^{\prime}$ is isomorphic to $U \oplus E_{6}(-1)^{2} \oplus A_{2}(-1)$ and its transcendebtal lattice is isomorphic to $A_{2} \oplus A_{2}(-1)^{\oplus 2}$. Thus the period domain of $K 3$ surfaces $S^{\prime}$ is a subdomain of $\mathcal{B}$ the orthogonal complement of $A_{2}(-1)$, that is, a $(-1)$-Heegner divisor. Thus we have 
SEGRE CUBIC

3.10. Proposition. $15(-1)$-Heegner divisors bijectively correspond to 15 planes on the Segre cubic $X$.

Proof. It is known that 15 planes on the Segre cubic correspond to the moduli of 6 points on the projective line in which two points coincide ([13], Proposition 3.2.7). Hence we have the assertion.

We shall show that $15(-2)$-Heegner divisors correspond to 15 Cayley cubics on the Segre cubic $X$ (see Lemma 6.6).

\section{WEIL REPRESENTATION}

In this section, we recall a representation of $\operatorname{SL}(2, \mathbf{Z})$ on the group ring $\mathbf{C}\left[A_{L}\right]$ called the Weil representation. In following Table 1 , for each vector $u \in A_{L}$ of given type, $m_{j}$ is the number of vectors $v$ of the same type with $\langle u, v\rangle=2 j / 3$.

$\begin{array}{rllllllllllllllll}u & 00 & 00 & 00 & 00 & 0 & 0 & 0 & 0 & 1 & 1 & 1 & 1 & 2 & 2 & 2 & 2 \\ v & 00 & 0 & 1 & 2 & 00 & 0 & 1 & 2 & 00 & 0 & 1 & 2 & 00 & 0 & 1 & 2 \\ m_{0} & 1 & 20 & 30 & 30 & 1 & 2 & 12 & 12 & 1 & 8 & 12 & 6 & 1 & 8 & 6 & 12 \\ m_{1} & 0 & 0 & 0 & 0 & 0 & 9 & 9 & 9 & 0 & 6 & 9 & 12 & 0 & 6 & 12 & 9 \\ m_{2} & 0 & 0 & 0 & 0 & 0 & 9 & 9 & 9 & 0 & 6 & 9 & 12 & 0 & 6 & 12 & 9\end{array}$

TABLE 1.

Let $T=\left(\begin{array}{ll}1 & 1 \\ 0 & 1\end{array}\right), S=\left(\begin{array}{cc}0 & -1 \\ 1 & 0\end{array}\right)$ be a generator of $\operatorname{SL}(2, \mathbf{Z})$. Let $\rho$ be the Weil representation of $\operatorname{SL}(2, \mathbf{Z})$ on $\mathbf{C}\left[A_{L}\right]$ defined by:

$$
\rho(T)\left(e_{\alpha}\right)=\exp (\langle\alpha, \alpha\rangle / 2) e_{\alpha}, \rho(S)\left(e_{\alpha}\right)=\frac{-1}{\sqrt{\left|A_{L}\right|}} \sum_{\delta} \exp (-\langle\delta, \alpha\rangle) e_{\delta} .
$$

Note that the action $\rho$ factorizes the action of $\operatorname{SL}(2, \mathbf{Z} / 3 \mathbf{Z})$ which is denoted by the same symbol $\rho$. The conjugate classes of $\operatorname{SL}(2, \mathbf{Z} / 3 \mathbf{Z})$ consist of $\pm E, S, \pm S T, \pm S T^{2}$. Let $\chi_{i}(1 \leq i \leq 7)$ be the characters of irreducible representations of $\operatorname{SL}(2, \mathbf{Z} / 3 \mathbf{Z})$. The following Table 2 is the character table of $\operatorname{SL}(2, \mathbf{Z} / 3 \mathbf{Z})$. Here $\omega=\frac{-1+\sqrt{-3}}{2}$ and the last line means the number of elements in a given conjugate class.

4.1. Lemma. Let $\chi$ be the character of the representation of $S L(2, \mathbf{Z} / 3 \mathbf{Z})$ on $\mathbf{C}\left[A_{L}\right]$. Let $\chi=$ $\sum_{i} m_{i} \chi_{i}$ be the decomposition into irreducible characters. Then $m_{1}=1, m_{2}=10, m_{3}=m_{4}=$ $5, m_{5}=5, m_{6}=10, m_{7}=5$.

Proof. By definition of $\rho$ and the Table 1 , we can easily see that $\operatorname{trace}(E)=3^{4}$, $\operatorname{trace}(-E)=1$, $\operatorname{trace}(S)=1, \operatorname{trace}\left(S T^{2}\right)=-9, \operatorname{trace}\left(-S T^{2}\right)=1, \operatorname{trace}(S T)=1, \operatorname{trace}(-S T)=-9$. The assertion now follows from the Table 2 . 


$\begin{array}{llllllll} & E & -E & S & S T^{2} & -S T^{2} & S T & -S T \\ \chi_{1} & 1 & 1 & 1 & 1 & 1 & 1 & 1 \\ \chi_{2} & 3 & 3 & -1 & 0 & 0 & 0 & 0 \\ \chi_{3} & 1 & 1 & 1 & \omega^{2} & \omega^{2} & \omega & \omega \\ \chi_{4} & 1 & 1 & 1 & \omega & \omega & \omega^{2} & \omega^{2} \\ \chi_{5} & 2 & -2 & 0 & -\omega & \omega & \omega^{2} & -\omega^{2} \\ \chi_{6} & 2 & -2 & 0 & -1 & 1 & 1 & -1 \\ \chi_{7} & 2 & -2 & 0 & -\omega^{2} & \omega^{2} & \omega & -\omega \\ & 1 & 1 & 6 & 4 & 4 & 4 & 4\end{array}$

TABLE 2.

4.2. Definition. Let $V$ be the 5-dimensional subspace of $\mathbf{C}\left[A_{L}\right]$ which is the direct sum of irreducible representations of $S L\left(2, \mathbf{F}_{3}\right)$ with the character $\chi_{3}$ in Lemma 4.1. In $₫ 6$, we associate a 5-dimensional space of automorphic forms on $\mathcal{B}$ to $V$. We remark that there is an another 5-dimensional subspace in $\mathbf{C}\left[A_{L}\right]$ which is a direct sum of irreducible representations of $S L\left(2, \mathbf{F}_{3}\right)$ with the character $\chi_{4}$. The author does not know whether this subspace corresponds to an interesting linear system of automorphic forms on $\mathcal{B}$.

\section{BORCHERDS PRODUCTS}

Borcherds products are automorphic forms on $\mathcal{D}$ whose zeros and poles lie on Heegner divisors. In this section, we shall show that there exist automorphic forms $\Phi_{45}, \Phi_{5}$ on the complex ball $\mathcal{B}(\subset \mathcal{D})$ of weight 45,5 whose zero divisors are (-2)-Heegnear divisor, $(-1)$ - Heegnear divisor respectively.

To show the existence of such Borcherds products, we introduce the obstruction space consisting of all vector valued elliptic modular forms $\left\{f_{\alpha}\right\}_{\alpha \in A_{L}}$ of weight $(2+6) / 2=4$ and with respect to the dual representation $\rho^{*}$ of $\rho$ :

$$
f_{\alpha}(\tau+1)=e^{-\pi \sqrt{-1}\langle\alpha, \alpha\rangle} f_{\alpha}(\tau), \quad f_{\alpha}(-1 / \tau)=-\frac{\tau^{4}}{9} \sum_{\beta} e^{2 \pi \sqrt{-1}\langle\alpha, \beta\rangle} f_{\beta}(\tau) .
$$

We shall apply the next theorem to show the existence of some Borcherds products.

5.1. Theorem. (Borcherds [6], Freitag [9], Theorem 5.2) A linear combination

$$
\sum_{\alpha \in A_{L}, n<0} c_{\alpha, n} \mathcal{D}_{\alpha, n}, c_{\alpha, n} \in \mathbf{Z}
$$

of Heegner divisors is the divisor of an automorphic form on $\mathcal{D}$ of weight $k$ iffor every cusp form

$$
f=\left\{f_{\alpha}(\tau)\right\}_{\alpha \in A_{L}}, f_{\alpha}(\tau)=\sum_{n \in \mathbf{Q}} a_{\alpha, n} e^{2 \pi \sqrt{-1} n \tau}
$$

in the obstruction space, the relation

$$
\sum_{\alpha \in A_{L}, n<0} a_{\alpha,-n / 2} c_{\alpha, n}=0
$$


holds. In this case the weight $k$ is given by

$$
k=\sum_{\alpha \in A_{L}, n \in \mathbf{Z}} b_{\alpha, n / 2} c_{\alpha,-n}
$$

where $b_{\alpha, n}$ are the Fourier coefficients of the Eisenstein series in the obstruction space with the constant term $b_{0,0}=-1 / 2$ and $b_{\alpha, 0}=0$ for $\alpha \neq 0$.

In the following we shall study the divisors $\sum_{\alpha \in A_{L}, n<0} c_{\alpha, n} \mathcal{D}_{\alpha, n}$ where $c_{\alpha, n}$ depends only on the type of $\alpha$. Recall that there are 1, 20, 30 and 30 elements in $A_{L}$ of types (00), (0), (1) and (2), respectively (see Lemma 3.5). We denote the vector valued modular form $\left(f_{\alpha}\right)_{\alpha \in A_{L}}$ by

$$
\left(f_{00}, f_{0}, f_{1}, f_{2}\right)
$$

where each $f_{t}$ is the sum of the $f_{\alpha}$ as $\alpha$ varies over the elements of $A_{L}$ of type $t$. A calculation shows that the action of a generator $\{S, T\}$ of $\operatorname{SL}(2, \mathbf{Z})$ with respect to this basis is given by

$$
\rho^{*}(T)=\left(\begin{array}{cccc}
1 & 0 & 0 & 0 \\
0 & 1 & 0 & 0 \\
0 & 0 & \omega^{2} & 0 \\
0 & 0 & 0 & \omega
\end{array}\right), \quad \rho^{*}(S)=\frac{-1}{9}\left(\begin{array}{cccc}
1 & 1 & 1 & 1 \\
20 & -7 & 2 & 2 \\
30 & 3 & 3 & -6 \\
30 & 3 & -6 & 3
\end{array}\right) .
$$

5.2. Lemma. The dimension of the space of modular forms of weight $4=(2+6) / 2$ and of type $\rho^{*}$ is 2. The dimension of the space of Eisenstein forms of weight 4 and of type $\rho^{*}$ is also 2.

Proof. The dimension is given by

$$
d+d k / 12-\alpha\left(e^{\pi \sqrt{-1} k / 2} \rho^{*}(S)\right)-\alpha\left(\left(e^{\pi \sqrt{-1} k / 3} \rho^{*}(S T)\right)^{-1}\right)-\alpha\left(\rho^{*}(T)\right)
$$

([6], section 4, [9], Proposition 2.1). Here $k=4$ is the weight,

$$
d=\operatorname{dim}\left\{x \in V: \rho^{*}(-E) x=(-1)^{k} x\right\}=4
$$

and

$$
\alpha(A)=\sum_{\lambda} \alpha
$$

where $\lambda$ runs through all eigenvalues of $A$ and $\lambda=e^{2 \pi \sqrt{-1} \alpha}, 0 \leq \alpha<1$. A direct calculation shows that

$$
\alpha\left(e^{\pi \sqrt{-1} k / 2} \rho^{*}(S)\right)=1, \alpha\left(\left(e^{\pi \sqrt{-1} k / 3} \rho^{*}(S T)\right)^{-1}\right)=4 / 3 \text { and } \alpha\left(\rho^{*}(T)\right)=1 .
$$

On the other hand, the space of Eisenstein series is isomorphic to the subspace of $V$ given by

$$
\rho^{*}(T)(x)=x, \rho^{*}(-E)(x)=(-1)^{k} x
$$

(see Remark 2.2 in [9]). Thus we have the assertion.

Next we shall calculate a basis of Eisenstein forms of weight 4 and of type $\rho^{*}$. Let

$$
E_{1}=G_{4}(\tau, 0,1 ; 3), E_{2}=G_{4}(\tau, 1,0 ; 3), E_{3}=G_{4}(\tau, 1,1 ; 3), E_{4}=G_{4}(\tau, 1,2 ; 3)
$$

be Eisenstein series of weight 4 and level 3 (see [15], Chap. III, $\S 3$ ). Then the action of $S, T$ is as follows:

$$
T: E_{2} \rightarrow E_{3} \rightarrow E_{4} \rightarrow E_{2},
$$

$T$ fixes $E_{1}$, and $S$ switches $E_{1}$ and $E_{2}, E_{3}$ and $E_{4}$ respectively. 
Now we can easily see that a basis of Eisenstein forms of weight 4 and of type $\rho^{*}$ is given by

$$
\begin{gathered}
f_{00}=a E_{1}+b\left(E_{2}+E_{3}+E_{4}\right), \\
f_{0}=(-a-9 b) E_{1}+(-3 a-7 b)\left(E_{2}+E_{3}+E_{4}\right), \\
f_{1}=(-3 a+3 b)\left(E_{2}+\omega E_{3}+\omega^{2} E_{4}\right), \\
f_{2}=(-3 a+3 b)\left(E_{2}+\omega^{2} E_{3}+\omega E_{4}\right),
\end{gathered}
$$

where $a, b$ are parameters. The Fourier expansions of $E_{i}$ are given as follows (see [15], Chap. III, $\S 3$, Proposition 22):

$$
\begin{gathered}
E_{1}=\frac{(2 \pi)^{4}}{2 \cdot 3^{6}}+c\left(-3^{3} q+\cdots\right), \\
E_{2}=c\left(q^{1 / 3}+\left(2^{3}+1\right) q^{2 / 3}+3^{3} q+\cdots\right), \\
E_{3}=c\left(\omega q^{1 / 3}+\left(2^{3}+1\right) \omega^{2} q^{2 / 3}+3^{3} q+\cdots\right), \\
E_{4}=c\left(\omega^{2} q^{1 / 3}+\left(2^{3}+1\right) \omega q^{2 / 3}+3^{3} q+\cdots\right),
\end{gathered}
$$

where $c=\frac{(-2 \pi \sqrt{-1})^{4}}{3^{4} 3 !}=\frac{(2 \pi)^{4}}{2 \cdot 3^{5}}$. Put $a=-\frac{3^{6}}{(2 \pi)^{4}}$ and $a=-9 b$. Then

$$
\begin{gathered}
f_{00}=-1 / 2+2 \cdot 3^{3} q+\cdots, \\
f_{0}=10 \cdot 3^{3} q+\cdots, \\
f_{1}=135 q^{2 / 3}+\cdots \\
f_{2}=15 q^{1 / 3}+\cdots
\end{gathered}
$$

It follows from Lemma 5.2 that there are no non-zero cusp forms in the obstruction space. Hence Theorem 5.1 implies that

5.3. Theorem. There exist automorphic forms on $\mathcal{D}$ of weight 135,15 with some character whose zero divisors are (-4/3)-Heegnear divisor, (-2/3)-Heegnear divisor, respectively.

Since $(-4 / 3)-,(-2 / 3)$-Heegnear divisors meet the complex ball with multiplicity 3 (Lemma 3.8), we can take the cube root of these automorphic forms and then we have:

5.4. Corollary. There exist automorphic forms $\Phi_{45}, \Phi_{5}$ on the complex ball $\mathcal{B}$ of weight 45,5 whose zero divisors are (-2)-Heegnear divisor, (-1)- Heegnear divisor. 


\section{GRITSENKO-BORCHERDS LIFTINGS}

In this section, by applying the theory of liftings [5], we construct a linear system of automorphic forms on $\mathcal{B}$ with respect to $\Gamma(\sqrt{-3})$ which gives a birational map from the Segre cubic to the Igusa quartic.

Let $\rho$ be the Weil representation given in $\S 4$. A holomorphic map

$$
f: H^{+} \rightarrow \mathbf{C}\left[A_{L}\right]
$$

is called a vector valued modular form of weight $k$ and of type $\rho$ if

$$
f(M \tau)=\rho(M)(c \tau+d)^{k} f(\tau)
$$

for any $M=\left(\begin{array}{ll}a & b \\ c & d\end{array}\right) \in \operatorname{SL}(2, \mathbf{Z})$. We shall construct a 5-dimensional space of vector valued modular forms of weight 4 and of type $\rho$.

Let $V$ be the 5-dimensional subspace of $\mathbf{C}\left[A_{L}\right]$ in Definition 4.2. First we shall consider the following special vectors $v_{\alpha_{0}}$ in $V$ (We remark that the following definition of $v_{\alpha_{0}}$ is similar to the one given in Allcock-Freitag [3] to construct liftings in their case). Let $\alpha_{0}, \alpha_{1}, \alpha_{2}, \alpha_{3}$ be an orthogonal basis of $A_{L}$ with $q_{L}\left(\alpha_{0}\right)=-4 / 3, q_{L}\left(\alpha_{1}\right)=q_{L}\left(\alpha_{2}\right)=q_{L}\left(\alpha_{3}\right)=-2 / 3$. If we take $\alpha_{0}$, then such basis is uniquely determined up to signs. For each such basis, we define a vector $v_{\alpha_{0}}=\left(c_{\alpha}\right)_{\alpha \in A_{L}}$ in $\mathbf{C}\left[A_{L}\right]$ as follows:

$$
c_{\alpha}=1,0,-1
$$

according to

$$
\prod_{i}\left\langle\alpha, \alpha_{i}\right\rangle=1,0,-1 \in \mathbf{F}_{3}
$$

For example, assume $\alpha_{0}=(1,0,0,0), \alpha_{1}=(0,1,0,0), \alpha_{2}=(0,0,1,0), \alpha_{3}=(0,0,0,1) \in A_{L}=$ $\left(\mathbf{F}_{3}\right)^{4}$. Then $c_{\alpha} \neq 0$ if and only if $\alpha \in\{( \pm 1, \pm 1, \pm 1, \pm 1)\}$.

6.1. Lemma. Let $v_{\alpha_{0}}=\left(c_{\alpha}\right)$ be as above. Then $\rho(S)\left(v_{\alpha_{0}}\right)=v_{\alpha_{0}}, \rho(T)\left(v_{\alpha_{0}}\right)=\omega v_{\alpha_{0}}$. Moreover $r_{\alpha_{i}}\left(v_{\alpha_{0}}\right)=-v_{\alpha_{0}}$ for the reflection $r_{\alpha_{i}}$ associated with $\alpha_{i}$.

Proof. It suffices to prove the case $\alpha_{0}=(1,0,0,0), \alpha_{1}=(0,1,0,0), \alpha_{2}=(0,0,1,0), \alpha_{3}=$ $(0,0,0,1)$. Let $M=\{( \pm 1, \pm 1, \pm 1, \pm 1)\}$. Then $v_{\alpha_{0}}=\sum_{\alpha \in M} c_{\alpha} e_{\alpha}$. If $c_{\alpha} \neq 0$, then $q_{L}(\alpha)=-4 / 3$. Hence $\rho(T)\left(v_{\alpha_{0}}\right)=\omega v_{\alpha_{0}}$. Next consider

$$
\rho(S) v_{\alpha_{0}}=-\frac{1}{9} \sum_{\beta \in A_{L}}\left(\sum_{\alpha \in M} c_{\alpha} e^{-2 \pi \sqrt{-1}\langle\alpha, \beta\rangle}\right) e_{\beta} .
$$

A direct calculation shows that the coefficient

$$
\sum_{\alpha \in M} c_{\alpha} e^{-2 \pi \sqrt{-1}\langle\alpha, \beta\rangle}
$$

of $e_{\beta}$ is 0 if $\beta \notin M, 9$ if $\beta \in M, c_{\beta}=-1$, and -9 if $\beta \in M, c_{\beta}=1$. The last assertion follows from the definition of $v_{\alpha_{0}}$.

It follows from Lemma 6.1 that $v_{\alpha_{0}}$ is contained in $V$. Thus we have fifteen elements $v_{\alpha_{0}}$ in $V$ where $\alpha_{0}$ is fifteen $(-4 / 3)$-vectors in $A_{L} /\{ \pm 1\}$. 
6.2. Lemma. As a $O\left(q_{L}\right)$ module, $V$ is irreducible.

Proof. If $W$ is a irreducible representation of $\mathfrak{S}_{6}$ and $\operatorname{dim} W \geq 2$, then $\operatorname{dim} W \geq 5$. Hence it suffices to see that there are no 1-dimensional subspaces invariant under the action of $\mathfrak{S}_{6}$. If such 1 -dimensional subspace exists, then all vectors in $V$ are invariant under the action of $\mathfrak{S}_{6}$. However any special vector $v_{\alpha_{0}}$ as above is not invariant under the action of $\mathfrak{S}_{6}$. This is a contradiction.

Let $\eta(\tau)$ be the Dedekind eta function. Then

$$
\begin{aligned}
& \eta(\tau+1)^{8}=\omega \cdot \eta(\tau)^{8} \\
& \eta(-1 / \tau)^{8}=\tau^{4} \cdot \eta(\tau) .
\end{aligned}
$$

Therefore, for $v \in V, \eta(\tau)^{8} \cdot v=\left(\eta(\tau)^{8} \cdot c_{\alpha}\right)_{\alpha \in A_{L}}$ is a vector valued modular form of weight 4 and of type $\rho$. By applying Gritsenko-Borcherds lifting ([5], Theorem 14.3), we have

6.3. Lemma. There is a 5-dimensional space of holomorphic automorphic forms of weight 6 on $\mathcal{D}$ with respect to $\tilde{O}(L)$ on which $O\left(q_{L}\right)$ acts irreducibly.

Proof. It suffices to see that the lifting of $\eta(\tau)^{8} v$ is non-zero. Then the assertion follows from the Schur's lemma. We use Theorem 14.3 in [5]. We consider the orthogonal decomposition of $L=$ $U \oplus U(3) \oplus A_{2} \oplus A_{2}$. Let $z, z^{\prime}$ be a basis of $U$ with $z^{2}=z^{\prime 2}=0,\left\langle z, z^{\prime}\right\rangle=1$, and let $K=z^{\perp} / \mathbf{Z} z=$ $U(3) \oplus A_{2} \oplus A_{2} \subset L$. Let $e, f$ be a basis of $U(3)$ with $e^{2}=f^{2}=0,\langle e, f\rangle=3$. We consider the Fourier expansion around $z$. Since $\eta(\tau)^{8}=q^{1 / 3}+\cdots$, the initial term of

$$
\eta(\tau)^{8} v=\sum_{\alpha \in A_{L}} e_{\alpha} \sum_{n \in \mathbf{Q}} c_{\alpha}(n) e^{2 \pi \sqrt{-1} n \tau}
$$

is

$$
\sum_{\alpha \in A_{L}, \alpha^{2}=2 / 3} c_{\alpha} q^{1 / 3}
$$

If we take $\lambda=(e+f) / 3$, then $\langle\lambda, \lambda\rangle=2 / 3>0$ and hence $\lambda$ has positive inner products with all elements in the interior of the Weyl chamber. Also note that $L^{*} / L=K^{*} / K$. We choose $v=\left(c_{\alpha}\right) \in$ $V$ satisfying $c_{\lambda} \neq 0$. Now it follows from [5], Theorem 14.3 that the Fourier coefficient of $e^{2 \pi \sqrt{-1}\langle\lambda, Z\rangle}$ in the lifting of $\eta(\tau)^{8} v$ is equal to

$$
c_{\lambda}\left(\lambda^{2} / 2\right) \cdot e^{2 \pi \sqrt{-1}\left\langle\lambda, z^{\prime}\right\rangle}=c_{\lambda}(1 / 3)=c_{\lambda} .
$$

Hence the lifting of $\eta(\tau)^{8} v$ is non-zero.

Let $\alpha_{0}$ be a $(-4 / 3)$-vector in $A_{L}$ and let $v_{\alpha_{0}}$ be the element in $V$ as above. Let $F_{\alpha_{0}}$ be the restriction of the Gritsenko-Borcherds lifting of $\eta(\tau)^{8} \cdot v_{\alpha_{0}}$ to the complex ball $\mathcal{B}$. Then

6.4. Theorem. $F_{\alpha_{0}}$ is a holomorphic automorphic form of weight 6 on $\mathcal{B}$ with respect to $\Gamma(\sqrt{-3})$ which vanishes exactly along the (-2)-Heegner divisor $\mathcal{H}_{\alpha_{0}}$ with multiplicity one and the $(-1)$ Heegner divisors $\mathcal{H}_{\alpha_{1}}, \mathcal{H}_{\alpha_{2}}, \mathcal{H}_{\alpha_{3}}$ with multiplicity three.

Proof. First recall that the reflection $r_{\alpha}$ is induced from the reflection $r_{a,-1}$ of $\Lambda$ where $a \in \Lambda$ is a short or long root with $a \bmod \sqrt{-3} \Lambda=\alpha$. It follows from Lemma 6.1 and the $\mathrm{O}\left(q_{L}\right)$-equivariance of the lifting that $F_{\alpha_{0}}$ vanishes along $\mathcal{H}_{\alpha_{i}}(i=0,1,2,3)$. Moreover the trireflection $r_{a, \omega}$ associated to a short root $a$ is contained in $\Gamma(\sqrt{-3}), F_{\alpha_{0}}$ vanishes along $\mathcal{H}_{\alpha_{i}}(i=1,2,3)$ with multiplicity 3 . Then the product of $15 F_{\alpha_{0}}$ has weight 90 and vanishes along (-2)-Heegner divisors with at least 
multiplicity one and along $(-1)$-Heegner divisors with at least multiplicity $\frac{3 \cdot 15 \cdot 3}{15}=9$. On the other hand $\Phi_{45} \cdot \Phi_{5}^{9}$ has weight 90 and vanishes along $(-2)$-Heegnear divisors with exactly multiplicity one and along $(-1)$-Heegnear divisors with multiplicity 9 (Corollary 5.4). Then the ratio $\prod_{v} F_{v} / \Phi_{45} \cdot \Phi_{5}^{9}$ has weight zero and holomorphic, and hence it is constant by Koecher principle.

Since trireflections are contained in $\Gamma(\sqrt{-3})$, the covering $\mathcal{B} \rightarrow \mathcal{B} / \Gamma(\sqrt{-3})$ is ramified along $(-2 / 3)$-Heegnear divisors. Hence we have

6.5. Theorem. The zero divisor $\left(F_{\alpha_{0}}\right)$ on $\overline{\mathcal{B}} / \Gamma(\sqrt{-3})$ is $\overline{\mathcal{H}}_{\alpha_{0}}+\overline{\mathcal{H}}_{\alpha_{1}}+\overline{\mathcal{H}}_{\alpha_{2}}+\overline{\mathcal{H}}_{\alpha_{3}}$.

6.6. Lemma. Let $\alpha \in A_{L}$ with $q_{L}(\alpha)=-4 / 3$. Then the Heegner divisor $\overline{\mathcal{H}}_{\alpha}$ coincides with a Cayley cubic on $X$.

Proof. The zero divisor of $\Phi_{45}$ on $\overline{\mathcal{B}} / \Gamma(\sqrt{-3})$ is the union of $\overline{\mathcal{H}}_{\alpha}$ where $\alpha$ varies over $15(-4 / 3)$ vectors in $A_{L} /\{ \pm 1\}$. On the other hand, as mentioned as above, the covering $\mathcal{B} \rightarrow \mathcal{B} / \Gamma(\sqrt{-3})$ is ramified along (-1)-Heegnear divisors. Hence the zero divisors of $\Phi_{5}^{3}$ on $\overline{\mathcal{B}} / \Gamma(\sqrt{-3})$ is the union of $\overline{\mathcal{H}}_{\alpha}$ where $\alpha$ varies over $15(-2 / 3)$-vectors in $A_{L} /\{ \pm 1\}$. If $\alpha_{1}, \alpha_{2}, \alpha_{3}$ are mutually orthogonal $(-2 / 3)$-vectors, then $\overline{\mathcal{H}}_{\alpha_{1}}+\overline{\mathcal{H}}_{\alpha_{2}}+\overline{\mathcal{H}}_{\alpha_{3}}$ is the union of three planes on $X$ (Proposition 3.10 ), that is, a linear section of $X$ in $\mathbf{P}^{4}$. By comparing the weights of $\Phi_{45}$ and $\Phi_{5}^{3}$, we can see that each $\overline{\mathcal{H}}_{\alpha}$ with $q_{L}(\alpha)=-4 / 3$ is also a linear section, that is, a cubic surface. Since $\overline{\mathcal{H}}_{\alpha}$ with $q_{L}(\alpha)=-4 / 3$ contains four cusps, it should be isomorphic to a Cayley cubic on $X$.

Hence we conclude:

6.7. Lemma. The divisor $\left(F_{\alpha_{0}}\right)=\overline{\mathcal{H}}_{\alpha_{0}}+\overline{\mathcal{H}}_{\alpha_{1}}+\overline{\mathcal{H}}_{\alpha_{2}}+\overline{\mathcal{H}}_{\alpha_{3}}$ is a quadric section of the Segre cubic $X \subset \mathbf{P}^{4}$ where $\overline{\mathcal{H}}_{\alpha_{0}}$ is a Cayley cubic and $\overline{\mathcal{H}}_{\alpha_{i}}(i=1,2,3)$ are planes.

For an orthogonal basis $\left\{\alpha_{0}, \alpha_{1}, \alpha_{2}, \alpha_{3}\right\}$ of $A_{L}$, we can easily see that any isotropic vector in $A_{L}$ is perpendicular to $\alpha_{i}$ for some $i$ (see Lemma 3.5). Hence the divisor $\overline{\mathcal{H}}_{\alpha_{0}}+\overline{\mathcal{H}}_{\alpha_{1}}+\overline{\mathcal{H}}_{\alpha_{2}}+\overline{\mathcal{H}}_{\alpha_{3}}$ contains 10 nodes of $X$. Thus each $\left(F_{\alpha_{0}}\right)$ passes the ten nodes of $X$, and hence the five dimensional linear system of automorphic forms has the ten nodes as base points. The linear system defines a rational $\operatorname{map} \varphi: X--\rightarrow \mathbf{P}^{5}$.

6.8. Theorem. The image of $\varphi$ is the Igusa quartic, that is the dual of $X$.

Proof. This follows from [13], Theorem 3.3.12.

\section{REFERENCES}

[1] D. Allcock, New complex and quaternion-hyperbolic reflection groups, Duke. Math. J., 103 (2000), 303-333.

[2] D. Allcock, J. A. Carlson, D. Toledo, The Complex Hyperbolic Geometry of the Moduli Space of Cubic Surfaces, J. Algebraic Geometry, 11 (2002), 659-724.

[3] D. Allcock, E. Freitag, Cubic surfaces and Borcherds products, Comm. Math. Helv., 77 (2002), $270-296$.

[4] H. F. Baker, Principles of Geometry, Vol. IV, Cambridge University Press 1925.

[5] R. Borcherds, Automorphic forms with singularities on Grassmannians, Invent. Math. 132 (1998), $491-562$.

[6] R. Borcherds, The Gross-Kohnen-Zagier theorem in higher dimensions, Duke Math. J., 97 (1999), $219-233$.

[7] J. H. Conway et al., Atlas of Finite Groups, Oxford University Press, 1985.

[8] I. Dolgachev, B. van Geemen, S. Kondō, A complex ball uniformaization of the moduli space of cubic surfaces via periods ofK 3 surfaces, J. reine angew. Math., 588(2005), 99-148.

[9] E. Freitag, Some modular forms related to cubic surfaces, Kyungpook Math. J., 43 (2003), 433-462. 
[10] B. van Geemen, Projective models of Picard modular varieties, Classification of irregular varieties, Proceedings, Trento, 1990 (Ed. by E. Ballico), Lect. Notes in Math., 1515, Springer, Berlin 1992, 68-99.

[11] B. Hunt, A Siegel modular 3-fold that is a Picard modular 3-fold, Comp. Math., 76 (1990), $203-242$.

[12] B. Hunt, S. T. Weintraub, Janus-like algebraic varieties, J. Differential Geometry, 39 (1994), 509-557.

[13] B. Hunt, The geometry of some special arithmetic quotients, Lect. Notes in Math., 1637 (1996), Springer.

[14] J. Igusa, On Siegel modular forms of genus two, Amer. J. Math., 86 (1964), 219-245; 88(1964), $392-412$.

[15] N. Koblitz, Introduction to elliptic curves and modular forms, 2nd ed., Springer 1993.

Graduate School of Mathematics, Nagoya University, Nagoya, 464-8602, JaPAN

E-mail address: kondo@math. nagoya-u.ac.jp 